geschlagen, bisler leider obnc eiuen praktischen Erfolg. Kintweder man wollte in der Blase selbst den einen Ureter zudrïcken (Silbcrmann) oder durch zwei Hebel von Blase und Mastdarm ( $\mathrm{Polk}$ ), oder bloss vom Mastdarm mittels eines Ballons mit $\mathrm{Hg}$ resp. durch die Bauchwand mittels eincr Art Tourniquct (M üller, diese Wochenschr. 1887); S ands wollte einc Digitalcoulpression des Haruleiters vom Yastdarm ausführen. In neuester Zeit plaidirt Sänger und Hegar für die limstcchung eines Ureters von der Scheide aus und will sehr gute Resultate gesehen haben (Warkulla). Doch haben sich alle diese Methoden, auch die Sänger'sche, praktisch noch nicht genügend eingeführt; und soll man heute, wo alle diese Jaassnahmen erst in der Fntwickelung begriffen sind, sclion ein Urtheil abgchen, so wäre es dieses: Für den II anu ist bis jetzt dic sicherstc aber auch cingreifendste Methode das Vorgchen C $x$ crny's, einc Fistel anzulcgen und den Urin daraus zu erhalten zu suchen. Für die Frau wird man die simon'sche oder dic Simon-Pawlick'sche Methode versuchen und, wenu diesc misslingt, die Sänger'sche, endlich als letzte Zuflucht dic Nicrenbeckenfistel. Zu dieser letzteren Operation wird man sich aber in der Regel crst dam cntschliessen, wenn hinterher die Exstirpation der Niere in Frage kommt. Sonst muss man sich mit den spărlichen Mitteln für die l)iagnosc begnügen, und die Lectüre der Krankengcschichteu zeigt, dass heute das Schmerzgefühl dcs Patienteu für dic Annahmc einer rechts" oder linksseitigcu Nierenerkrankung meist ausschlaggebend ist. Freilich kann man durch das Tastgefühl Erstaunliches leisten, wie uns $1 \mathrm{srael}$ gezeigt hat. Es ist kaum glaublich, dass er einen Nierentumor von der Grössc einer halben Kirsche richtig palpirt und glücklich operirt hat, und es mag wohl richtig sein, was die älteren Aerzte uns jüngeren vorwerfen, dass wir vor allen complicirteren Untersuchungsmethoden (Hurchen und Klopfen) die cinfachere vcrnachlässigen. Lauenstcin mcint, wenn man eine Blase local-methodisch 5 bis

\section{Rückblicke auf die Chirurgie des letzten Jahres.}

Von Dr. Emil Senger in Berlin.

\author{
(Schluss aus No. 25.)
}

Wir kommen mu zu dem wicltigen Kapitel der Nierenchirurgie.

Wic weit sind wir hicr in cinem Zeitraum von 15 Jahren gckounnen, seit Simon 1869 die erste Ncphrectomie mit Krfolg untcrnommen hat! Es giebt heutc kcinen grössern Chirurgen, der nicht schon an diesem Organ operirt hätte; ja es ist noch nicht lange her, dass dic Chirurgic crbarmungslos viel weiter als nöthig ihr Gebiet ausdehnte und 2. B. die vicre nur wegen schlaffer Fixation etc. (Wandernierc) fortnahm. Der Gynäkologe Landau hat der Chirurgie einen grossen Dienst gelcistet, dass er ihr diese ludication zur Exstirpation entriss. Man hat an Stelle der letateren in dem Ictzten Jahre vielfach init angeblich gutem Erfolge die Anheftung der Niere nach Hahn (Nephrorrhaphie) ausgeführt, obrohl geradc Landau das Prinzip der Methode als unrichtig erklärt. Die Bestrebungen von Landau, Nussbaum, Bandl, Schede u. A. im vergangenen Jahre gingen weiter dahin, auch die Ureterfistel nicht durch Nierenexstirpation zu beseitigen, sondern durch conservative II ethoden, wie z. B. durch Anfrischung und Naht über einem Kathetcr. Und doch ist bei diesen Leiden die Diagnose meist klar.

Dic Schwierigkeit der Nicrenchirurgie bcginut erst, Nenn es sich um Krankheiten im Organ handelt, un Steinc, Tumorell, Hydround Pyelonephrosen etc., weil wir in vielen Fällen mit unübcrwindlichen diagnostischen Schwierigkeiten zu kämpfen haben. Vor dem Gedanken an eine Nierenoperation muss erst eine Reihe von Fragen beantwortct scin. Welche Niere ist krank, in welchem functioncllen Zustand befindet sich die kranke, in w'clchem die gcsunde Nicre?

Die diagnostischen Hauptfragen wurden von vielcn Seiten und durch viele Mittel zu lösen gcsucht. Im Verhältniss zu denselben sind die auch im letzten Jahre zahlreich angegebenen Schnittführungcu nur von untergeordnetem Interesse. Ob man dell alten Simon'schen verticalen Lumbalschuitt oder den latcro-lumbalen oder deu transperitonealen (d. $h$. deu durch das Peritoncum hindurchführendeu) oder den retro- und intrapcritonealen (König) anwenden, ob man den Paticnten auf den Bauch oder auf die Seite mit oder ohne Unterschiebung eines Rollkissens legen solle, ist für die allgemeine Nierenchirurgie nebensächlich und erhält nur seine hohe Bedeutung durch den cinzclnen Fall. Wichtiger schou sind dic Untersuchungen le Dentu's, welche die Beziehung der Jctzten Rippe 2.0 der Pleura studiren und zeigten, dass man, am rertebralen kinde ciner Rippe operircnd, immer in Gefahr sei, die Pleura zu öfnen, am vorderen Viertheil dagegen ohne Gefahr operiren könne (Revue de chirurg. VI (1-2) 1886). Die grösste Wichtigkcit bchält immer die Diagnosc. Dic grössten Clirurgen haben sich darin geirrt, indem sic eine Ovarialcystc oder einen Echinococcus für eine Nierengeschwulst gehalten haben, und durch dicsen Irrthum ist sogar manchmal der Tod des Patienten verschuldet (Vergl. dic Lchrbücher von Olshausen und Spencer Wells 1886.)

Lelier wclche Mittel verfügen wir heute zur Stellung einer Diagnose?

Wir habcn eine ganze Reihe von Methoden, welche alle darauf hinauslaufen, das Secret einer Niere gesondert zu erhalten. Man hat drei Wege eingeschlagen: 1) das Katheterisircn des Ureters, 2) Anlcgung einer Fistel im Ureter odcr im Nicrenbecken, 3) temporären Verschluss des Ureters. Pawlick will die Katheterisation ohne Dilatation der Urethra vornehmen. Er giebt an, dass bei der Frau in Knieellenbogenlage das Trig. Lientandii durch Falten an der vorderen Vaginal wand markirt sei und das Kathetcrisiren leicht gclinge. Er soll sich in der That cine grosse Fertiglieit darin erwerben haben; aber leider lässt sich dicsc Methode nur bei lirauen anwenden. Für Männer wie auch für Frauen will Harrison resp. Emmet vorher dcn Perineal- rcsp. den Blasenscheidenschnitt machen und dann katheterisiren. Aber auch so ist dieses sehr schwierig wegen des Klappenventils des Ureters in der Blasc. Der dritte Wcg, $d$. h. einen V'erschluss des Ureters herbeizuführen, ist von einer Reihe von Autoren ein-
6 Wochen ohne jeden Erfolg behandelt habc, so könne man bei citerigem Urin auf eine Pyelitis schliessen. Wie wcit uns dic elektrischc Beleuchtuug der Blase mit der Diagnosc fördern wird, lässt sich noch gar nicht ermessen. - Bevor man aber an die Nephrectomic herangeht, muss man sich, für den Fall, dass dic zu entfernende Niere krank ist, noch die wich. tige Frage beantworten: In welchem functionellen Zustande befindet sich die audere zurükbleibende Niere? Zu diesem $/$ wecke muss man die Menge des Harnstoffes in 24 Stunden durch genaue chemische Analysc des vorsichtig aus der Fistel aufgcfangenen. meist mit etwas Eiter vermischten Urins bestimmen. Man muss wissen, wie viel Harnstoff von der

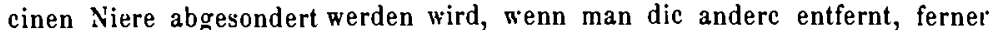
wic gross die Mengc des Harnstoffes sein müsse, damnit der Hicnsclı an Leben blcibe. Ohne die Kenntniss oder die annälıende Schätzung dieser Verhältnisse ist man nicht berechtigt, eine Exstirpation au vollführen. Ja, Israel betont sogar, dass man auch bci der genauesten Kenntniss der Harnstoffmenge leider erfahren müsse, dass man sich mit der Natur verrechnet habe, da nach ihn durch die Operation der cinen Nicrc cine rcflectorische Schwankung iu der Function der andcren cintreten könuc, so dass die zurückblcibende Niero vicl weniger sccernirt als vor der Operation n1ul als zum Leben nothwendig ist: währeud die relativ gesunde Nierc rorler ein Quantum Harnstoff ausgeschieden häte, welches noch sehr gut init dcm Leben vereinbar wäre, sei dasselbe nach der Operation so wcit gesunken, dass der Tod eintrat.

Kann es da Wunder uehmen, wenn einem Operateur ein Patient nach der Nephrectomie stirbt, bei dem die Nierenthătigkeit nicht vorher geprüft wurde, und bei dem sich nachher herausstellt, dass beidc Fieren eiterig oder cystisch entartct oder sonst schwer krank waren? Hier zeigt sich, und darum führte ich nur das obige an, wie ohnmächtig die Chirurgie, auf wie niedrigem Niveau sie steht, wenn sie nicht die Untcrsuchungsmethoden der iuneren Medicin, wie dio Chcrie etc. sie uns bieten, sich zu Nutzell macht und beherrscht, es zcigt sich, dass ein Fortschritt in unserer Wisseuschaft nur durch die fortschreitende kirweitcrung der Diagnose erreicht werden kann, nicht so schr durch die Tcchnik.

Eine wichtige Frage ist ferner folgende: Wonn man unit Sicherheit eincn Nierenstein erkannt hat, soll man die Nephrolithotomie machen, bevor Eiterung erfolgt ist? Gross hat statistisch (Med. News Dec. 1886) sich dahin entschieden, dass man vor der Eiterung operircn müsse, weil das Vorhandensein einer Pyelitis die Prognose bcdeutend verschlechtert. Es starben nămlich von 34 Operirten mit eiterlosen Steinnieren $3=8,8^{\circ} \%$, von 33 mit Pyelitis $11=33 \%$. Es giebt noch viele Fragen in der Nierenchirurgie, welche der Erledigung harren, aber wir haben schon den Raum für den Bcricht über Nierenchirurgie überschritten und wollen zu den Blasen- und Harnkrankhciten übergehen.

Am meistcn crörtert wurde wohl die Frage der Lithotomie, nicht nur in Deutschland, sondern anch in der Pariser chirurgischen Gesellschatt, der englischen Roy. chir. Society und in Russland. Trotzdem ist eine Einigung nicht darin crzielt, wann lithotripsirt, wann litholapaxirt, wann die Scctio alta mediana lateralis gcmacht werden solle. Obwohl Koraca. letzthin für die S. lateralis cingetrcten ist, ist sic doch fast allgcmein verworfen, und es trcten nur noch dic alta und die mediana in Concurrenz, und zwar so, dass die mediana als cinc riemlich ungefăhrliche Opcration für die meistcu Fälle geuügen dürftc, dass die alta dagegen wegen der Fistelbildungen, der Harniufiltration, Verletzung des Pcritoneum gefährlicher aber bci der Entfernung sehr grosser Stcinc leistungsfähiger sei. Die Gefahr der Fistclbildung will Brenner lurch eine ncue Blasennaht (nach Art der Tabaksbcutelschnur) abwenden, die Gcfahr der Peritonealverletzung aber lässt sich auch durch die Rectumtamponade nicht immer beseitigen, da in seltcneu Fällen das Peritoneum bis an die Symphyse reicht. Wiesinger will die Fistelbildungen durch Jodoformtamponade der Blase bei der $\mathrm{S}$. alta vermeiden. Dittel lehrte uns in einer interessanten Studie, dass eine Leichenblasc durch $300-1500 \mathrm{~g}$ Wasscr zum Bersten gebracht werden könnc, nachdem er selbst und Weinlechucr es erlebt hat, dass Kindern von 5 Jahren durch Injection vor der Sect. alta dic Blase geborsten ist. Dittel spricht sich dahergegen die Füllung der Blase aus. 
Bei der Ektopia vesicae kölnon wir einen grossen Fortschritt verzeichnen Schon Passavant (ja schon Demme 1855) suchte orthopädisch durch Zug und Druck dio Symphysen gleich nach der Geburt zu nälıern. I rendclenburg will diese länger daucrndc Behandlung eliirurgisch wit e in e m $\mathrm{M}$ a $\mathrm{l}$ c bewirken, indem er die Synchondr. sacro-iliac. trennt, die hintcrel Bänder durchschncidet und danı durch einen gekrcuzten und belasteten Gurt die Symphysen zusammendrängt. Nach 4-6 Wochen crfolgt die Auffrischung und Silberdraht-Naht. Natürlich lässt sich diese Methode bloss bei Kindern bis 7u 5-6 Jahren anwenden, wo las Becken noch biegsam ist. Bei älteren bleibt nur die Thiersch'sche Iransplantation übrig.

In der Diagnose der Blasenkranklıeiten scheint durch las elektrische Cystoskop einc neue Aera angebahnt zu sein. Nitze will eine Reihe pathologischer Zustände, Divertikel, Tumoren etc. richtig erkannt habcn. Hoffen wir, dass die bis jetzt noch nicht zu entbehrende Simousche Ilcthode, die bimanuclle Untersuchung etc. durch die mildere Uroskopie ganz verdrängt wird. Freilich erfordert die Untersuchung mit dca Nit $z$ eschen Instrument, wic ich mich sclbst überzcugen inusstc, Uebung und Technik, wic ctwa das Ophthalmoskop. Das Instrument ist auch sehr theucr unt wohl auch noch verbesserungsfähig.

Posncr hat das Grūnfeld'sche Endoskop für die Harnröhre dahin modificirt, dass er die fiüherc mattc Inncufläche durch hellspicgelnde Flächen (amalgamirte Glasröhre, dic aussen schwarz lackirt ist) ersctz.t.

Wenn ich die Urethra noch mit in den Bereich der chirurgischen Betrachtungen ziehc, olwohl ich weiss, dass dicses Gebiet von den Specialisten als ilure Domäne betrachtct wird, so möchte ich crwähnen, dass Unna uns seine Methode der Behandlung der Gonorrhoe gelehrt hat; sic besteht darin, durch einc mit einer passenden Salbe bestrichene Sondc zu gleicher Zeit einen Druck auszuübcu und local zu behanclch. (Therap. Honatsl. 1887). Casper hat einc Modification derselben, seinc canellirte Sonde, l. h. zu deutsch gerillte oder mit Rinmen verschenc Sonde" nachdrücklich empfohlen. Telt?. hat balıl daraut cine Mlodification der Modification angegeben, und ein Andercr cine andere Salbenform als wirksamer gcrühmt. Kintgegen dicsen Bestrebungen nehmen sehr viele $\Lambda$ crate von der Salbensondenbchandlung $\ddot{b}$ berhaupt $A$ bstand. krebs.

Doch gchen wir zu eincm ernsteren Lciden über: zu dem Mastuarm-

Wälırend die Englïnder und Franmosen eine Exstirpation resp. Resection höchst selten ansführen und lieber einc Colotomie machell, gehen dic deutschen Chirurgen radicaler vor. Aber auch bci diescn scliwanken die Grenzen für die Zulässigkeit der Uperation; $\mathrm{K}$ önig will für hoch oben gclegenc Carcinome keinc Exstirpation, sondern nur die Colotomie ausfüluren, und in der That können die so behandciten Patienten noch Jahre lang bei bestem Wohlsein sich befinden. - Bardenhcucr aber kennt keine Grenzen für dic Indication der Radicaloperation, mag die Geschwulst nach unten oder wcit naclı oben gewuchert sein. Fr liat ¿. B. bei eincr 80jährigen Frau ein $40 \mathrm{~cm}$ langes Rectumstück resecirt, will nur $10 \%$ Mortalität bei complicirteren, $5 \%$ bei lcichteren, uncouplicirten Fällen haben. Er durchschneidet 7.war das Lig. tub.-sacr, und spinoso.-sacr. und durchtrennt ziemlich hoch das Os sacrum bis zum 3. Kreuzbeinloch, will aber stumpf rorgehen und hat nur 2-4 Ligaturen nöthig, wo wir sonst $50-60$ brauchten, hat nur 15-30 Min. Operationsdauer, wo wir sonst $2-3$ Stunden nöthig hatten. Ob diese frappirenden Resultate bei der obigen Methode auch von andereu Chirurgen errcicht werden, wird die nächstc Zeit lehren: sie würden eincn grossen Fortschritt in der I'echnik bedeuten; aber die meisten Operateure dürftcı nohl hente für allzuhohe Carcinome mit König die Colotomie vorriehen. (Volkm. Vortrag 1887 No. 298).

Für die $H a ̈$ anorrhoiden lıat Lange nach amerikanischem Hhıstcr eine Carbol-Glycerininjection ohne Narkose cmpfohlen, hochliegendc Fisteln will er excidiren. In der Lehre von den II ernicn hat uns Küster die bisher nicht bekannten Herniae inguino-supcrficiales kemnen gelehrt, d. h. jenc Hernien, wo das Bauchfell unter der Haut des Bauches, Dammes und des Oberschenkcls sich befindet.

Anderegg (Basel) sucht in einer grossen Arbeit zu zeigen, dass mit oder ohne Pfortcnnaht, mit oder ohuc Bruchband der Erfolg der Herniotomie in Berug auf das Recidiv gleich sci; bei wachscnden Individucn sei das Recidiv riel seltencr.

Mit einigen Worten wollen wir noch das Gcbiet der inneren Eink lemmungen, welches auf lem vorletztcn Chirurgencongress so eingehend erörtert wurde, streifen.

Von den grossen Fortschritten der (hirurgie ist die Lehre von den innereu Einklemmungen nicht betroffen: dic Leute sterben heute rerade so oft, wie zur Zeit Dieffenbach's. Auch hier zeigt sich wieder. wie schwer wir die geringe Sicherheit in der Iiagnose empfinden. Wir erkennen meist erst cine Finklemmung, wenn der Collaps bercits eingetroten ist. Dann aber nützt meist die Operation nichts, da dicsc den Collaps nicht bckämpfon kann, sondern vielmehr vergrössert und den l'od herbeiführt. Dic Prognose der inneren Incarcerationen wird sich also erst dann bessern, wenn wir eine sichcre Diagnose vordemeingetrctcnen Collaps stellen könucn. So lange wir das nicht könuen, licgt der Hauptfortschritt in der Erkenntniss der Crsachc des Collapses und wie dieser hintanzuhalten resp. zu bescitigen ist. $O b$ der Collaps durch Intoxication mit Fäulnissalkaloiden cntstelıe, ob er rein ncrvöser Natur sei, soll die

1) Iclı möchte mir lier nur dic Bemerkung erlauben, dass mir der deutsche Ausdruck viel schöner und verständlicher klingt und auch richtiger scheint Denn obwohl man \%. B. von canellirten Säulcn, Canellirungsmaschinen der Büchsenmacher spricht, so kommt das Wort vou canna das Rohr, cannula das Röhrchen, Stöckchen her, woher auch unsere bekannte "Canüle*. In unserem medicinischen Sprachgebrauch würde dann also "canellirte Sonden heissen: "geröhrte Sonden", was doch niclıt den thatsächlılichen Bcgriff deckt.
Forschung reigen. Heute sterben die Leute am Collaps geradc so, wic sie früher nach IIerniotomieen an Peritonitis zu Grunde gingen. Viele Operateure wollen nicht früh operiren, weil erfahrungsgcmäss sclbst die ver\%weifeltsten Fällc spontan naclı 25 ja nach 35 Tagen wieder heilen können. Curschmann einpfiehlt allc 3-4 Stunden rom Darm aus eine Lufteinblasung, besonders bei tief unten liegender Ursachc; sodann dic Darmpunction zum Zwecke der Gascntlecrung, wcil dic Aufblälıung des l)arms ein küıstliches Klappenventil schaffe. Auf die alten Wasscreinläufe vorichtet Curschmann. Ol) abcr dic Chirurgen sich zur Darinpunction entschliessen nicrden, ist fraglich; die meisten habcu heute vielmehr wohl folgende Mcinung: In den meisten Fällen wird dic wcuig gefährliche Enterostomie zn machen sein, wohei es schwicrig ist, die Darmschlinge oberhalb des Verschlusses zu fasscı. Dic rothcongestionirten, stärker venös injicirten Darmthcile, die intensivcre Peristaltik, eine durch dic Bauchdccke lcisc markirte Darınschlinge (Fuhr) wcrden ein Merkival abgeben. Nachdem sich der Paticut erholt hat, kann man die Radicaloperation vornehmen. Ist der Collaps klein oder noclı gar nicht da, ist der Sitz und die Art der Finklemmung klar, so wird inan lap:rotomiren und sofort las Hinderniss bescitigen können.

Eng an dicses Capitel schlicsiren sich dic Laparotomieen wegen eitriger. und jauchiger Peritonitis. Von 4 Patienten sind (Poelchen) 3 gestorben. Antiscptische Bauchausspülungen scheinen ohne besondcrn Nutzen zu scin. mehr Vortheile schcint:n Jodotorıgaz:ausstopfungen und Drainage zu versprechen.

Nicht 7.u erktären, aber dcnnoch sicher' constatirt sind die relativen Heilungen von $B$ : uchfelltulerculose nach Laparotomieen. Kümmel, Weinstein, Schwar\%, II ofmokl habcu uns derartigc Fäle mitgetheilt. Glcich\%eitig bestehende Jungenphthise wird durch rlie Japarotomic nicht bceinflusst oder gar gcheilt. lie Eutlcerung etwa bestehenden Ascites, div gesteigcrte Circulation, die antiseptische Ausspülung kanı nicht dic Besserung der Tuberculose bewirkc1. Zu vergesson ist nicht, dass vicle tuberculösc l'eritonitiden überhaupt sehr langsann verlaufen, und dass vielleicht die Hauptheschwerden nur von do:n Ascites herrühren.

Hier möchte ich in Kürze auf dic zalılrcichen Il agenoperationen hinweisen, wciche im vergangencn Jahrc gemacht sind. Wegen Fremdkörper sind von Polaillon, Crcde, Richardson, Bernays Gastrotomiccn ausgeführt worden. Polaillon wics den Sitz eines Mlessers durch die Abweichung ciner vor die Jlagengrube gchaltenen astatischen (elektromagnctisch(n) Nadel nach. Von 13 Operirten sind 2 gestorben; die Fremdkörper (Messcr, Gabcl, Ha:ıre etc.) wurden crst nach Iagen oder Jahren extrahirt. Ucber die Gastro-kntcrostomie liegt eine sorgfältige Arbeit von Rockwits vor (s. Z. f. Chir. Bd. XXV.), bei 21 Operirten hat er eine Mortalität von 570 . Dic Gefahr bei der Operation ist dic Cornpression des Colon durch dic hinübergeführte Dünnularmschlinge und das Vernähen des ungeknickten Iarmes. Nach ciner Pylorusrcsection kounte Wölfler nach fünf Jahren eine lïnstlich gebildcte Falte erkemucn, wclche den Pylorus darstclltc; Koch cr dagegen sah eine Stcnose cntstehen. -

Dass auch Ocsophagotomicen, ja Excisionen des Oesophagus mit plastischem Verschluss aus llautstücken vorgenommen wurden (Alsberg, Mikulic\%), dürftc zu bekannt sein. - Bemerkenswerth ist noch eine durch Küster richtig crkannte und glücklich operirte Pankreascyste. Die Diagnose dieser Gebilde ist nicht lcicht. Die Cysten sind verwechselt worden mit Aneurysmen, wit Echinococeen der Bursa omentalis, Cysten der Nebenniere. Die letztere konnte Küster wegen der Grösse der fraglichen Geschrulst aussthciden, ein Ancurysma deshalb, weil eine Vcrmehrung der Spanuung nach der Seite hin nicht eintrat; die Punctionsflüssigkeit nar eiwcisshaltig, und es blieb wegen des Sitzes nur eine Pankrcascyste übrig. J)ieselbe besitzt das Saccharific ıtionsvermügcn und die Fähigkeit, Fette $2 . u$ emulgiren, also dem Pankreassaft zukonımende Eigenscliaften. Au dicse Pankreascyste lässt sich am besten die Operation einer Chyluscyste aus der $r$. Bergmann'schen Klinik anschliessen. -

Wenn uns zum Schluss noch eine Bcmerkung zur Gynaeknlogie gc stattct ist, so möchten wir anführen, dass die Ovarialtumoren in etwa $22,4 \%$ nach Schultzc und ca. 16,6\% nach Cohn (Schröder) malign werden, und dass man daher einer frühzeitigen Operation der Ovarialtunoreu das Wort reden muss. Hinsichtlich des Carcinoms der Portio scheinen heute wohl bis auf einzelnc Operateure (U of meier) alle die hohc Cervixamputation verlassen $\mathrm{zu}$ habcil und gleich die Totalcxstirpation vorzunehmen.

Damit wollen wir den Bcricht über die Leistungen der Chirurgie schliessen. Es sind vielfache Pfade und Wege chirurgischer Bcstrebungen, welche ich versucht habe, zu einew Gcsammtbilde - allerdings wohl zu eincm recht bunten - zu rereinigen und in einen gewissen Zusammenhang zu bringen. Und ronn wir die leitcnden Ideen in dicsem Bilde studiren, d. h. aus dem Gange der Chirurgie aus dem vorigen Jahre Schlüsse zichen wollen, so würden wir Folgendes lernen können: Gewiss wird man noch manche schönen Neuerungen und Fortschrittc in der Chirurgie der Knochen resp. der Extremitäten erreichen; aber die Hauntaufgabe für die nächsten Jahre ist die chirurgische Behandlung inncrcr lírankheiten, der Kanpf mit der innercn Mredicin. I)ie Chirurgie wird in diesem Kampfe nur dann ibrer Gegncrin gewachsen sein, wcnn sie sich der gleichen Waffen bedicnt; wenn sie die Untersuchungsmetholen der inmeren Jedicin anwendet und sich die Chemie, Physik, Bacteriologie etc. zu Nutze macht. Wir babcn oben gesehen, dass ein Fortschritt il dor Chirurgic des Gehirns, der Lcber, Niere etc. nur durch eine sicherere Diagnosc gewährlcistct wird. So segensrcich auch die Antiseptik nach verschiedenen Richtungen hin gewirkt hat, so hat sic doch dazu beigetragın, die geistige $H$ öhe der chirurgischen Diagnostik herabzudrücken. Denn wie oft haben wir es geschen, dass dort, wo der innere Mlediciner seincn Hammer, sein Gefühl, chemischc Kenntnisse, sodann logisch-wissen. schaftliche Combinationen anwendet, der kühne und glücklicl operirende Chirurg nur das Messer zur Diagnosostellung ergreift? Wie manche Probelaparotomie hätte bci Erschöpfung der Untersuchungsınittel unterbleiben künnen! Der Fortschritt der Chirurgie also liegt in der inneren Medicin 
und in deren Studiun. Der Unterschied, welcher Jahrhunderte lang
zwischen beiden Disciplinen der Medicin aufrecht erbalten wurde, wird ill eiuigeu Jahrzehnten _- das ist uusere Ueberueuguug - immer mehr verschwinden, und die Zeit liegt nicht fern, wo inan den Satz aussprechen darf: ein Chirurg inuss nicht nur eine gute Amputation und Resection machen können, sondern vor allen auch ein iunerer Mlediciner sein. 